\title{
DIFFERENTIABLE FUNCTIONS WHICH DO NOT SATISFY A UNIFORM LIPSCHITZ CONDITION OF ANY ORDER
}

\author{
MASAYOSHI HATA
}

(Communicated by R. Daniel Mauldin)

\begin{abstract}
The aim of this paper is to construct two kinds of absolutely continuous functions. One is differentiable everywhere but does not satisfy a uniform Lipschitz condition of any order on some large class of subintervals, while the other is differentiable almost everywhere but does not satisfy a uniform Lipschitz condition of any order on any subintervals.
\end{abstract}

\section{INTRODUCTION}

The importance of the distinction between a function that, at each point of an interval, satisfies some local Lipschitz condition and a function that satisfies a uniform Lipschitz condition throughout the interval was pointed out by $\mathrm{G}$. $\mathrm{H}$. Hardy [3]; indeed, he remarked that the function

$$
\sum_{n=1}^{\infty} n^{-7 / 8} \cos \left(2 n \pi x+n^{3 / 4}\right)
$$

satisfies a local Lipschitz condition of order 1 at every point, but it does not satisfy a uniform Lipschitz condition of any order greater than 1/4 throughout the interval $(0,1)$. In this respect, our first theorem provides more extreme examples. We denote by $|E|$ the usual Lebesgue measure of $E$ for any measurable subset $E \subset \mathbf{R}$.

Theorem 1.1. For any $\varepsilon \in(0,1)$, there exist a strictly increasing, absolutely continuous function $f_{\varepsilon}(x)$ defined on $[0,1]$ and a closed set $E_{\varepsilon} \subset[0,1]$ with $\left|E_{\varepsilon}\right| \geq 1-\varepsilon$ such that

(a) $f_{\varepsilon}(x)$ has a finite derivative at every point $x \in(0,1)$; and

(b) for any closed subinterval $J$ satisfying $\stackrel{\circ}{J} \cap E_{\varepsilon} \neq \varnothing$ and for any $\alpha \in$ $(0,1]$,

$$
\sup _{\substack{x \neq y \\ x, y \in J}} \frac{\left|f_{\varepsilon}(x)-f_{\varepsilon}(y)\right|}{|x-y|^{\alpha}}=\infty .
$$

(In particular, this holds for every interval $J$ satisfying $|J| \geq \varepsilon$.)

Received by the editors October 31, 1989.

1980 Mathematics Subject Classification (1985 Revision). Primary 26A16; Secondary 26A27.

Key words and phrases. Lipschitz conditions, discontinuous derivatives. 
In the above theorem, every point of $E_{\varepsilon}$ must be a discontinuity point of the derivative $f_{\varepsilon}^{\prime}(x)$, for otherwise, $f_{\varepsilon}(x)$ would satisfy a uniform Lipschitz condition of order 1 on some small interval $J$ with $J \cap E_{\varepsilon} \neq \varnothing$, from the mean value theorem. Thus $E_{\varepsilon}$ can not contain any interval, since $f_{\varepsilon}^{\prime}(x)$ is continuous except at a set of first category, by Baire's theorem on functions of first class; hence, $E_{\varepsilon}$ must be nowhere dense.

We also note that the classical examples of everywhere differentiable, nowhere monotone functions (see, for example, E. W. Hobson [5], Y. Katznelson and K. Stromberg [6]) satisfy a uniform Lipschitz condition of order 1 on any interval (more precisely, the derivatives are uniformly bounded).

Now let $N(f) \subset[0,1]$ be the set of points of nondifferentiability of $f(x)$ for any continuous function $f(x)$ defined on $[0,1]$. If $f(x)$ does not satisfy a uniform Lipschitz condition on any subinterval, then the set $N(f)$ must be large in the sense of category. More precisely, we have

Lemma 1.2. Let $f(x)$ be any continuous function defined on $[0,1]$ which does not satisfy a uniform Lipschitz condition of order 1 on any subinterval. Then $N(f)$ is a residual set (in particular, $N(f)$ is dense and uncountable).

The first example of continuous functions which do not satisfy a uniform Lipschitz condition of any order on any interval was obtained by G. Faber [2]; indeed, he constructed a continuous function $g(x)$ satisfying $g(x+h)-g(x) \neq$ $O\left(\left.|\log | h\right|^{-1}\right)$ as $h \rightarrow 0$ at every point $x$. Such an example was also found by G. H. Hardy [3], who showed that the function

$$
g(x)=\sum_{n=1}^{\infty} n^{-2} \cos \left(b^{n} \pi x\right)
$$

satisfies $g(x+h)-g(x) \neq o\left(\left.|\log | h\right|^{-2}\right)$ as $h \rightarrow 0$ at every point $x$ if $b>1$. So these classical nowhere-differentiable functions provide examples of Lemma 1.2 with $N(g)=[0,1]$.

On the other hand, we can construct a strictly increasing continuous function $g(x)$ satisfying $g(x+h)-g(x) \neq o\left(\left.|\log | h\right|^{-2}\right)$ as $h \rightarrow 0$ at every point $x=q \cdot 2^{-p} \in(0,1)$ for positive integers $p, q$ using Salem's geometrical construction [8]. Such a function can also be defined as a unique continuous solution $g(x)$ of a system of infinite difference equations; for example,

$$
(n+2)^{2} g\left(\frac{2 i+1}{2^{n+1}}\right)=(2 n+3) g\left(\frac{i}{2^{n}}\right)+(n+1)^{2} g\left(\frac{i+1}{2^{n}}\right),
$$

for $0 \leq i \leq 2^{n}-1, n \geq 0$ with boundary conditions $g(0)=0, g(1)=1$ (see M. Hata and M. Yamaguti [4]). Note that the solution $g(x)$ is purely singular; that is, $g^{\prime}(x)=0$ almost everywhere. Since $\left\{q \cdot 2^{-p} \in(0,1) ; p, q \in \mathbf{N}\right\}$ is a dense subset of $[0,1]$, this provides an example of Lemma 1.2 with $|N(g)|=0$. 
In this respect we have a more extreme example, as follows:

Theorem 1.3. There exists a strictly increasing, absolutely continuous function $h(x)$ defined on $[0,1]$ such that

(a) the Hausdorff dimension of the set $N(h)$ is zero; and

(b) for any subinterval $J$ and for any $\alpha \in(0,1]$,

$$
\sup _{\substack{x \neq y \\ x, y \in J}} \frac{|h(x)-h(y)|}{|x-y|^{\alpha}}=\infty .
$$

It is well known that if $f(x)$ satisfies a uniform Lipschitz condition of order 1 on an interval, then it is absolutely continuous. The converse is false, and our function $h(x)$ in Theorem 1.3 gives an extreme counterexample; in fact, $h(x)$ is absolutely continuous but does not satisfy any Lipschitz condition on any subinterval.

A. S. Besicovitch and H. D. Ursell [1] showed that if $f(x)$ satisfies a uniform Lipschitz condition of order $\alpha \in(0,1]$ on an interval, then the Hausdorff dimension of the graph of $f(x)$ on the interval is less than or equal to $2-\alpha$. Moreover, they showed that the converse is false by constructing a continuous curve $g_{\delta}(x)$ for each $\delta \in[1,2-\alpha]$ satisfying exactly a uniform Lipschitz condition of order $\alpha \in(0,1)$, for which the dimension $\delta$ is actually attained. In this respect, the function $h(x)$ in Theorem 1.3 provides a more extreme counterexample; in fact, the graph of $h(x)$ has a finite 1-dimensional Hausdorff measure (indeed, it is rectifiable, since $h(x)$ is of bounded variation), while $h(x)$ does not satisfy any Lipschitz condition on any subinterval.

\section{Preliminaries}

Our method of construction of the peculiar functions specified in Theorems 1.1 and 1.3 is similar to that of Y. Katznelson and K. Stromberg [6], by which they have constructed an everywhere-differentiable but nowhere-monotone function. In this paper we use the following peaked functions

$$
\phi_{n}(x) \equiv(1+|x|)^{(1-n) / n}
$$

for $n \in \mathbf{N}$ as basic functions, though Katznelson and Stromberg used only $\phi_{2}(x)$ as a basic function in their construction.

For any $n \in \mathbf{N}$ let $K_{n}(\mathbf{R})$ be the set of all positive continuous functions $f(x)$ defined on $\mathbf{R}$ such that

$$
\frac{1}{x-y} \int_{y}^{x} f(s) d s \leq 2 n \cdot \min \{f(x), f(y)\}
$$

for any $x \neq y \in \mathbf{R}$. Then it is easily seen that $\phi_{n}(x) \in K_{n}(\mathbf{R})$ for all $n \geq 1$. Note that (2.1) forbids $f(x)$ decreasing so rapidly as $|x| \rightarrow \infty$; in fact, it can be seen that

$$
\lim _{|x| \rightarrow \infty} \frac{|x|}{(\log |x|)^{m}} f(x)=\infty
$$

for any $m \in \mathbf{N}$. For example, the function $\phi_{\infty}(x) \equiv(1+|x|)^{-1}$ does not belong to any $K_{n}(\mathbf{R})$. One can easily verify the following lemma by simple calculation. 
Lemma 2.1. (a) $K_{n}(\mathbf{R})$ is a positive cone; that is, $\sigma_{1} f_{1}(x)+\sigma_{2} f_{2}(x) \in K_{n}(\mathbf{R})$ for any $f_{i}(x) \in K_{n}(\mathbf{R})$ and for any $\sigma_{i}>0 \quad(i=1,2)$.

(b) $K_{n}(\mathbf{R})$ is invariant under linear transformations of the variable; that is, $f(\tau(x-\beta)) \in K_{n}(\mathbf{R})$ for any $f(x) \in K_{n}(\mathbf{R})$ and for any $\tau>0, \beta \in \mathbf{R}$.

We now give the following generalization of Lemma 4 in [6], which is a crucial lemma in this paper.

Lemma 2.2. Suppose that $\psi_{n}(x) \in K_{n}(\mathbf{R})$ for all $n \geq 1$ and that

$$
\sum_{n=1}^{\infty} n \psi_{n}(c)<\infty
$$

for some $c \in \mathbf{R}$. Put $\Psi_{n}(x)=\int_{0}^{x} \psi_{n}(s) d s$ for brevity. Then the series

$$
\Lambda(x)=\sum_{n=1}^{\infty} \Psi_{n}(x)
$$

converges uniformly on any compact subset of $\mathbf{R}$. Moreover $\Lambda(x)$ is strictly increasing and absolutely continuous and has a finite derivative at $x=c$; in fact,

$$
\Lambda^{\prime}(c)=\sum_{n=1}^{\infty} \psi_{n}(c)
$$

Proof. Since $\psi_{n}(x) \in K_{n}(\mathbf{R})$, we have

$$
\left|\Psi_{n}(x)\right| \leq\left|\int_{0}^{c} \psi_{n}(s) d s\right|+\left|\int_{c}^{x} \psi_{n}(s) d s\right| \leq 2 n(|c|+|x-c|) \psi_{n}(c) .
$$

This implies the uniform convergence of $\sum_{n=1}^{\infty} \Psi_{n}(x)$ on any compact subset, which is clearly strictly increasing. Then

$$
\int_{0}^{x} \sum_{n=1}^{\infty} \psi_{n}(s) d s=\sum_{n=1}^{\infty} \int_{0}^{x} \psi_{n}(s) d s=\Lambda(x)
$$

for any $x \in \mathbf{R}$; in particular, $\sum_{n=1}^{\infty} \psi_{n}(x)$ is locally integrable, and $\Lambda(x)$ is absolutely continuous. Although it can be seen that $\Lambda^{\prime}(x)=\sum_{n=1}^{\infty} \psi_{n}(x)$ almost everywhere, we can show, as follows, that $\Lambda(x)$ is, in fact, differentiable at $x=c$.

For any $\varepsilon>0$, let $N \equiv N(\varepsilon)$ be a sufficiently large integer satisfying $\sum_{n>N} n \psi_{n}(c)<\varepsilon / 6$. Then there exists a sufficiently small $\delta>0$ such that

$$
\left|\frac{1}{h} \int_{c}^{c+h} \psi_{n}(s) d s-\psi_{n}(c)\right|<\frac{\varepsilon}{2 N}
$$

for any $0<|h|<\delta$ and any $1 \leq n \leq N$. Therefore,

$$
\begin{aligned}
\left|\frac{\Lambda(c+h)-\Lambda(c)}{h}-\sum_{n=1}^{\infty} \psi_{n}(c)\right| & \leq \sum_{n=1}^{\infty}\left|\frac{1}{h} \int_{c}^{c+h} \psi_{n}(s) d s-\psi_{n}(c)\right| \\
& \leq \frac{\varepsilon}{2}+\sum_{n>N}(2 n+1) \psi_{n}(c)<\varepsilon
\end{aligned}
$$


for any $0<|h|<\delta$. This completes the proof.

Let $\left\{m_{n}\right\}$ be a sequence of positive integers satisfying $m_{n}=O(n !)$ as $n \rightarrow$ $\infty$, and let $0 \leq \beta_{j, n} \leq 1$ for $1 \leq j \leq m_{n}, n \geq 1$. We then define the following closed set:

$$
L_{n}=\left\{x \in[0,1] ;\left|x-\beta_{j, n}\right| \geq \frac{1}{n !} \text { for all } 1 \leq j \leq m_{n}\right\}
$$

for any $n \geq 1$, and denote by $L$ the lower limit set of $\left\{L_{n}\right\}$; that is,

$$
L=\bigcup_{n=1}^{\infty} \bigcap_{k=n}^{\infty} L_{k} .
$$

We also denote by $D$ the derived set of $\left\{\beta_{j, n}\right\}$; in other words, $D$ is the set of all accumulation points of $\left\{\beta_{j, n}\right\}$. Clearly, $L$ is an $F_{\sigma}$-set, and $D$ is a closed subset of $[0,1]$. Then we have

Lemma 2.3. Put

$$
\psi_{n}(x)=(n !)^{n} \sum_{j=1}^{m_{n}} \phi_{n}\left((n !)^{n+4}\left(x-\beta_{j, n}\right)\right)
$$

for any $n \geq 1$. Then the function $\Lambda(x)$ appearing in Lemma 2.2 satisfies the following:

(a) $\Lambda(x)$ has a finite derivative at every point of $L$; and

(b) for any subinterval $J \subset[0,1]$ satisfying $\stackrel{\circ}{J} \cap D \neq \varnothing$ and for any $\alpha \in$ $(0,1]$,

$$
\sup _{\substack{x \neq y \\ x, y \in J}} \frac{|\Lambda(x)-\Lambda(y)|}{|x-y|^{\alpha}}=\infty .
$$

Proof. By Lemma 2.1, we have $\psi_{n}(x) \in K_{n}(\mathbf{R})$ for all $n \geq 1$. In general, $\psi_{n}(x)$ has $m_{n}$ sharp peaks (local maxima) of almost equal heights $(n !)^{n}$ at $x=\beta_{j, n}, 1 \leq j \leq m_{n}$. For any $x \in L$, there exists an integer $n_{x}$ such that $\left|x-\beta_{j, n}\right| \geq(n !)^{-1}$ for all $1 \leq j \leq m_{n}$ and all $n \geq n_{x}$. Then, clearly,

$$
\psi_{n}(x) \leq m_{n}(n !)^{n-(n+3)(n-1) / n}=m_{n}(n !)^{(3-2 n) / n} ;
$$

hence $\sum_{n=1}^{\infty} n \psi_{n}(x)<\infty$. Thus $\Lambda^{\prime}(x)=\sum_{n=1}^{\infty} \psi_{n}(x)$, by Lemma 2.2 .

Now suppose, on the contrary, that there exist an interval $J \subset[0,1]$ with $\stackrel{\circ}{J} \cap D \neq \varnothing, \alpha \in(0,1]$, and a positive constant $C$ such that $|\Lambda(x)-\Lambda(y)| \leq$ $C|x-y|^{\alpha}$ for any $x, y \in J$. Then we have

$$
J \supset\left[\beta_{j, N}, \beta_{j, N}+(N !)^{-N-4}\right] \equiv\left[\beta_{j, N}, \beta_{j, N}^{*}\right], \text { say, }
$$


for a sufficiently large integer $N$ and for some $j \in\left[1, m_{N}\right]$. We can here assume that $(N !)^{\alpha(N+4)-4}>2 C$. Then

$$
\begin{aligned}
C(N !)^{-\alpha(N+4)} & \geq \Lambda\left(\beta_{j, N}^{*}\right)-\Lambda\left(\beta_{j, N}\right)>\Psi_{N}\left(\beta_{j, N}^{*}\right)-\Psi_{N}\left(\beta_{j, N}\right) \\
& =\int_{\beta_{j, N}}^{\beta_{j, N}^{*}} \psi_{N}(s) d s>(N !)^{N} \int_{\beta_{j, N}}^{\beta_{j, N}^{*}} \phi_{N}\left((N !)^{N+4}\left(s-\beta_{j, N}\right)\right) d s \\
& =(N !)^{-4} \int_{0}^{1} \phi_{N}(t) d t>(N !)^{-4} \int_{0}^{1} \frac{d t}{1+t}>C(N !)^{-\alpha(N+4)} .
\end{aligned}
$$

This contradiction completes the proof.

\section{Proofs of Theorems 1.1 AND 1.3}

By virtue of Lemma 2.3, the proof of Theorem 1.1 can be reduced to the construction of a sequence $\left\{\beta_{j, n}\right\} \subset[0,1]$ such that $L=[0,1]$ and $|D| \geq 1-\varepsilon$ for an arbitrarily fixed $\varepsilon \in(0,1)$. Note that if $L=[0,1]$, then, by Lemma $1.2, D$ must be nowhere dense, so Theorem 1.1 says that $D$ can be large in the sense of measure while it is small in the sense of category.

Similarly, for the proof of Theorem 1.3, it suffices to construct a sequence $\left\{\beta_{j, n}\right\} \subset[0,1]$ such that $D=[0,1]$ and the Hausdorff dimension of the set $[0,1] \backslash L$ is zero. Note that if $D=[0,1]$, then $[0,1] \backslash L$ must be a residual set by Lemma 1.2 , since $[0,1] \backslash L \supset N(\Lambda)$; so Theorem 1.3 says that $[0,1] \backslash L$ can be small in the sense of Hausdorff measure while it is large in the sense of category.

We now give the proof of Theorem 1.1. For an arbitrarily fixed $\varepsilon \in(0,1)$ let $\lambda>0$ be a sufficiently small number satisfying $(1-3 \lambda) /(1-2 \lambda) \geq 1-\varepsilon$. We then define a Cantor set $E_{\varepsilon}$, obtained from $[0,1]$ by a sequence of deletions of open intervals known as middle thirds, as follows: First remove from $[0,1]$ the open interval $I_{1,1}$ centered at $\beta_{1,1}=1 / 2$ and of length $\lambda$. Let $\beta_{1,2}$ and $\beta_{2,2}$ be the middle points of the two remaining closed intervals, ordered from left to right, and remove from them the middle open intervals $I_{1,2}$ and $I_{2,2}$ of length $\lambda^{2}$, leaving four closed intervals, each of length $\left(1-\lambda-2 \lambda^{2}\right) / 4$. This process is permitted to continue indefinitely. At the $n$th stage of deletion, each length of the $m_{n} \equiv 2^{n-1}$ open intervals $I_{j, n}$ is $\lambda^{n}$ and each middle point of $I_{j, n}$ is denoted by $\beta_{j, n}$. The set $E_{\varepsilon}$ is defined to be the closed set remaining; thus we have $\left|E_{\varepsilon}\right|=(1-3 \lambda) /(1-2 \lambda) \geq 1-\varepsilon$.

By the construction, we have $\operatorname{dist}\left(E_{\varepsilon}, \beta_{j, n}\right)=\left|I_{j, n}\right| / 2=\lambda^{n} / 2$ for any $j$, $n$. Thus, obviously, $D=E_{\varepsilon}$ and $E_{\varepsilon} \subset L_{N}$ for all sufficiently large $N$; hence $E_{\varepsilon} \subset L$. Moreover, for an arbitrarily fixed open interval $I_{j, n}$, we have $I_{k, N} \cap$ $I_{j, n}=\varnothing$ for any $k$ and any $N>n ;$ hence $\operatorname{dist}\left(I_{j, n}, \beta_{k, N}\right) \geq\left|I_{k, N}\right| / 2=\lambda^{N} / 2$. Therefore $I_{j, n} \subset L_{N}$ for all sufficiently large $N$; so $I_{j, n} \subset L$. This means that $L=[0,1]$, as required. This completes the proof of Theorem 1.1.

We next give the proof of Theorem 1.3. For any $n \in \mathbf{N}$ let $\left\{\beta_{j, n}\right\}$ be the 
rational points expressed in the form $p / 2^{n}$, ordered from left to right, where $1 \leq p<2^{n}$ is an odd integer; so $m_{n} \equiv 2^{n-1}$. Since $\left\{\beta_{j, n}\right\}$ is a dense subset of $[0,1]$, we have $D=[0,1]$. By the definition of lower limit sets, for any $x \in[0,1] \backslash L$, there exists a strictly increasing subsequence $\left\{n_{i}\right\} \subset \mathbf{N}$ such that $x \notin L_{n_{i}}$ for all $i \geq 1$; that is,

$$
\left|x-\frac{p_{i}}{2^{n_{i}}}\right|<\frac{1}{n_{i} !}
$$

for some odd integer $p_{i}$. Thus the inequality $|x-p / q|<q^{-\xi}$ has an infinite number of integer solutions $p, q$ with $(p, q)=1$ for each fixed $\xi>0$, since $2^{\xi n}=o(n !)$ as $n \rightarrow \infty$. Therefore, $x$ is a Liouville number. On the other hand, the Hausdorff dimension of the set of Liouville numbers is zero (see, for example, J. C. Oxtoby [7, Theorem 2.4]). This completes the proof of Theorem 1.3 .

We do not know whether (1.1) and (1.2) in our theorems can be replaced by the following stronger property:

$$
\sup _{\substack{x \neq y \\ x, y \in J}}|\log | x-y|| \cdot|f(x)-f(y)|=\infty
$$

\section{Proof of Lemma 1.2}

In this section we give the proof of Lemma 1.2 in a standard way.

Let $f(x)$ be a continuous function, defined on $[0,1]$, which does not satisfy a uniform Lipschitz condition of order 1 on any subinterval of $[0,1]$. Put

$$
U_{n}=\left\{x \in(0,1) ; \sup _{x<y \leq 1} \frac{|f(y)-f(x)|}{y-x}>n\right\}
$$

for any $n \in \mathbf{N}$. Clearly $U_{n}$ is a dense subset of $[0,1]$ for each $n$. By the definition, for any $x \in U_{n}$, there exists a point $y_{0} \in(x, 1]$ satisfying

$$
\left|f\left(y_{0}\right)-f(x)\right|>n\left(y_{0}-x\right) \text {. }
$$

Thus the set $U_{n}$ is open, since this property is stable under a small perturbation on $x$. Hence the set $N(f)$ must be a residual set, since

$$
N(f) \supset\left\{x \in(0,1) ;\left|D^{+} f(x)\right|+\left|D_{+} f(x)\right|=\infty\right\}=\bigcap_{n=1}^{\infty} U_{n},
$$

where we denote, as usual, the upper and lower derivatives at $x$ of $f(x)$ on the right by $D^{+} f(x), D_{+} f(x)$ respectively. This completes the proof.

\section{ACKNOWLEDGMENT}

This work was inspired by a question which Prof. S. Okada (University of Tasmania, Australia) asked the author recently. The author also thanks the referee for his suggestions, which have made this paper easier to read. 


\section{REFERENCES}

1. A. S. Besicovitch and H. D. Ursell, Sets of fractional dimensions (V): On dimensional numbers of some continuous curves, J. London Math. Soc. 12 (1937), 18-25.

2. G. Faber, Einfaches Beispiel einer stetigen nirgends differentiierbaren Funktion, Jber. Deutsch. Math. Verein. 16 (1907), 538-540.

3. G. H. Hardy, Weierstrass's non-differentiable function, Trans. Amer. Math. Soc. 17 (1916), 301-325.

4. M. Hata and M. Yamaguti, The Takagi function and its generalization, Japan J. Appl. Math. 1 (1984), 183-199.

5. E. W. Hobson, The theory of functions of a real variable II, "Jover, New York, 1957, pp. 412-421.

6. Y. Katznelson and K. Stromberg, Everywhere differentiable, nowhere monotone functions, Amer. Math. Monthly 81 (1974), 349-354.

7. J. C. Oxtoby, Measure and category, Springer-Verlag, New York, Heidelberg, and Berlin, 1971.

8. R. Salem, On some singular monotonic functions which are strictly increasing, Trans. Amer. Math. Soc. 53 (1943), 427-439.

Institute of Mathematics, Yoshida College, Kyoto University, Kyoto 606, Japan 